\title{
EP-74
}

\section{Recurrent hepatic encephalopathy caused by the persistent porto-systemic shunt in liver transplant recipients}

\author{
Jaryung HAN, Young Seok HAN*, Yoon Jin HWANG
}

Department of Surgery, Kyungpook National University Hospital, Kyungpook National University School of Medicine, Daegu, Korea

Introduction: Hepatic encephalopathy (HE) caused by porto-systemic shunt is one of the representative complications of liver cirrhosis, but $\mathrm{HE}$ is an infrequent condition after the full restoration of portal flow through liver transplantation, despite the persistence of porto-systemic shunts. We report two cases with a normal functioning liver graft and patent portal flow that developed recurrent HE after liver transplantation.

Methods: We reviewed the medical record the two cases, liver transplant recipient represented HE with stable graft function.

Results: The one case received a living donor liver transplantation (LDLT) with inferior vena cava (IVC) replacement because of Budd-Chiari Syndrome. Two months after LDLT, recurrent HE occurred despite of well-functioning liver graft and patent portal vein. An abdominal computed tomography scan (CT) showed large port-systemic collaterals between mesenteric vein and IVC. The other case transferred for the further evaluation of recurrent HE. She received a deceased donor liver transplantation, 9 years ago, and a HE with hyperammonemia was repeated, despite of normal liver function test. Persistent meso-caval collateral veins were identified on CT. After the closure of shunt by the interventional angiographic embolization, hyperammonemia and HE were completely resolved.

Conclusions: Persistent porto-systemic shunts in liver transplant recipients with stable graft function may be the cause of HE. Therefore, when liver transplant patient with stable graft function represents HE, a more thorough evaluation is needed to identify the existence of persistent porto-systemic shunts that are very rare condition. 